\title{
PERFIL MOTOR DE CRIANÇAS E ADOLESCENTES COM DEFICIÊNCIA MENTAL MODERADA ${ }^{+}$
}

\section{MOTOR PROFILE OF CHILDREN AND ADOLESCENTS WITH MODERATE METAL RETARDATION}

Samira Schultz Mansur* Adair José Marcon ${ }^{* *}$

\begin{abstract}
Mansur SS, Marcon AJ. Perfil motor de crianças e adolescentes com deficiência mental moderada. Rev Bras Crescimento Desenvolv Hum. 2006; 16(3):09-15.
\end{abstract}

\begin{abstract}
Resumo: O controle motor de deficientes mentais é alterado pela insuficiência de informações entre os comandos aferente e eferente. Este estudo teve como objetivo avaliar o perfil motor de crianças portadoras de deficiência mental de grau moderado. O grupo de estudo foi constituído por 20 crianças, ambos os sexos, entre 7 e 15 anos, alunos das APAEs dos municípios de Biguaçu e Palhoça/SC, em 2005. Para a coleta de dados foi utilizada a Escala de Desenvolvimento Motor "EDM". O tratamento estatístico foi realizado com o programa Epi Info 6.0. A todas as habilidades motoras avaliadas foi atribuído nível "muito inferior". O quociente da motricidade fina obteve o menor déficit, sendo que ao quociente da organização temporal atribuiu-se o maior déficit. Tais resultados mostraram a estreita relação entre o potencial cognitivo e a conduta motora de deficientes mentais de grau moderado. Os déficits funcionais observados entre deficientes mentais podem ser conseqüentes à inadequação de suas habilidades psicomotoras, interferindo no desenvolvimento cognitivo, afetivo e social.
\end{abstract}

Palavras-chave: Perfil motor. Crianças. Adolescentes. Deficiência mental moderada.

\section{INTRODUÇ $\tilde{A O}$}

A taxa de prevalência da deficiência mental (DM) é de $1 \%$ na população jovem ${ }^{1}, 2$ a $3 \%$ entre jovens e adultos ${ }^{2}$, encontrando-se estimativas de até $10 \%{ }^{3}$. Vale citar que o uso do termo deficiência mental está sendo questionado desde 2001 pela American Association of Mental Retardation, atribuindo-se outros similares como "inabilidade de aprendizado" ou "inabilidade intelectual", mais difundidos, respectivamente, na Austrália e no Japão ${ }^{4}$.

A DM pode se originar de um defeito no crescimento axonal, dendrítico ou da função sináptica ${ }^{2}$ sendo que as anormalidades dendríticas são seus indicativos anatômicos mais consisten- tes ${ }^{5}$. Outrossim, a remodelagem das sinapses e as alterações na forma e no número das espinhas dendríticas são a base anatômica do aprendizado e da memória ${ }^{5}$.

A percepção e habilidades viso-motoras e motoras globais estão intrinsecamente relacionadas com substratos neurais assim como a processos cognitivos ${ }^{6,7}$. Ressalta-se a significância do córtex pré-frontal no processamento de informações verbais, motoras e emocionais e a dependência entre o desenvolvimento cerebral e cognitivo ${ }^{8}$.

Os sistemas sensitivos e motores possuem células especializadas e distintas funcionalmente e isso permite uma maior velocidade de processamento de informações e adequação de respostas, essencial para funções cognitivas ${ }^{9}$.

\footnotetext{
Trabalho realizado nas Associação de Pais e Amigos dos Excepcionais (Apaes) dos municípios de Biguaçu e Palhoça/ SC.

* $\quad$ Mestre em Ciências do Movimento Humano (UDESC). Rua Antônia Alves, 59. Bairro: Itaguaçu. CEP: 88085/390. Florianópolis/SC.E-mail: samirasmansur@hotmail.com

** Especialista em Atividade Motora Adaptada (UNICAMP).
} 
Ocorre que, entre deficientes mentais, o dano neurológico limita a comunicação intercelular através das sinapses intervindo na transferência de informações pelo sistema nervoso e dificultando a atividade neuronal eficiente ${ }^{2,9,10}$.

O conhecimento de áreas motoras específicas de deficientes mentais, como a motricidade fina e global, equilíbrio, esquema corporal, organização espacial e temporal e lateralidade, permite identificar suas dificuldades motoras, planejar o trabalho intervencionista e otimizar sua psicomotricidade. Urge esclarecer que, para uma ação multiprofissional de sucesso nessa população, um dos pontos primordiais é sua avaliação motora freqüente a fim de manter-se a par do aprendizado e desenvolvimento motor e cognitivo desses sujeitos. É importante elucidar que o aprendizado e o desenvolvimento estão sendo extensamente considerados similares e inseparáveis no tangente às aquisições cognitivas e motoras ${ }^{11}$, sendo necessário observar testes motores específicos, com variados graus de complexidade, principalmente em casos com baixos quocientes de inteligência ${ }^{12}$.

Esta pesquisa objetivou avaliar o perfil motor de crianças e adolescentes com deficiência mental moderada, matriculados nas Associações de Pais e Amigos dos Excepcionais/APAEs dos municípios de Biguaçu e Palhoça/SC, em 2005.

\section{MÉTODO}

Esta pesquisa se caracterizou por ser descritiva-diagnóstica e de campo. A população $(n=113)$ foi constituída por alunos matriculados nas APAEs dos municípios de Biguaçu e Palhoça/SC, em 2005. O processo de amostragem foi não probabilístico do tipo intencional.

A amostra $(n=20)$ foi composta por crianças e adolescentes na faixa etária de 7 a 15 anos, ambos os sexos, com deficiência mental moderada. Foram excluídos do grupo amostral alunos sindrômicos, paralisados cerebrais e com algum comprometimento físico, auditivo e/ou visual interveniente na avaliação motora. Os deficientes mentais de intensidade leve e grave/profunda não fizeram parte da pesquisa pois estes não responderiam aos testes propostos e aqueles não freqüentavam as APAEs.

A classificação da DM esteve em concordância com o Diagnostic and statistical manual of mental disorders ${ }^{13}$, que correlaciona a intensidade dessa deficiência com o escore do quociente de inteligência (QI). Assim, as crianças com QI de 50-55 a 70 têm DM leve; as com QI de 35-40 a 50-55, DM moderada; àquelas com QI de 20-25 a 35-40, DM grave; e as com QI inferior a 20-25, DM profunda.

A avaliação do perfil motor foi realizada pelo uso da Escala de Desenvolvimento Motor "EDM"14. O exame compreende testes de dificuldade graduada, em diferentes estágios do desenvolvimento, na faixa etária de 2 a 11 anos, além de identificar a lateralidade. Avalia seqüencialmente a motricidade fina, motricidade global, equilíbrio, esquema corporal, organização espacial e organização temporal e fornece idade e quociente motor geral e idades e quocientes motores nas áreas específicas citadas.

Esclarece-se que a lateralidade pode ser classificada em destra completa, sinistra, indefinida ou cruzada. Entende-se por lateralidade cruzada quando há preferência no uso, a exemplo, da mão direita e olho esquerdo, ou seja, o indivíduo utiliza o segmento corporal, sensorial e neurológico (mão, pé, olho, ouvido e hemisfério cerebral) de maneira cruzada.

A coleta dos dados foi responsabilidade de um único pesquisador e realizada em sala da Apae na qual o aluno estava matriculado, em local ausente de estímulos externos que atrapalhassem sua atenção. Cada integrante da amostra foi avaliado individualmente durante 30 a 45 minutos, sendo que nesse momento permaneciam na sala de avaliação somente o aluno e o pesquisador.

Os valores numéricos dos quocientes motores foram relacionados em níveis da "EDM", que permitem classificar as habilidades estudadas em padrões: "muito superior (130 ou mais), superior (120-129), normal alto (110-119), normal médio (90-109), normal baixo (80-89), inferior (70-79) e muito inferior (69 ou menos)".

Para obtenção do valor mínimo, média e valor máximo utilizou-se o programa informático Epi-info-6.0. 
TABELA 1 - Variáveis motoras deficientes mentais de grau moderado $(\mathrm{n}=20)$, entre 7 e 15 anos, das APAEs dos municípios de Biguaçu e Palhoça/SC, em 2005.

\begin{tabular}{lccc}
\hline Variáveis & Valor Mínimo & Média & Valor Máximo \\
\hline Idade Cronológica & 91 & 161 & 180 \\
\hline Idades Motoras & & & 74 \\
\hline Idade Motora Geral & 22 & 45 & 78 \\
\hline Idade Motora da Motricidade Fina & 24 & 51 & 84 \\
\hline Idade Motora da Motricidade Global & 24 & 47 & 84 \\
\hline Idade Motora do Equilíbrio & 24 & 44 & 72 \\
\hline Idade Motora do Esquema Corporal & 24 & 42 & 96 \\
\hline Idade Motora da Organização Espacial & 36 & 47 & 72 \\
\hline Idade Motora da Organização Temporal & 24 & 41 & 44 \\
\hline Quocientes Motores & & & 61 \\
\hline Quociente Motor Geral & 17 & 30 & 52 \\
\hline Quociente Motor da Motricidade Fina & 14 & 34 & 52 \\
\hline Quociente Motor da Coordenação Global & 15 & 31 & 47 \\
\hline Quociente Motor do Equilíbrio & 13 & 29 & 57 \\
\hline Quociente Motor do Esquema Corporal & 13 & 28 & 51 \\
\hline Quociente Motor da Organização Espacial & 20 & 30 & 27 \\
\hline Quociente Motor da Organização Temporal & 13 & & \\
\hline
\end{tabular}

NOTA: A média e os valores máximo e mínimo da idade cronológica, idade motora geral e idades motoras específicas têm a unidade "meses", enquanto os quocientes motores valores absolutos.

\section{RESULTADOS}

\section{Desenvolvimento motor}

De acordo com a tabela 1 , verificou-se discrepância de aproximadamente 9,5 anos (116 meses) entre a média da idade cronológica (IC) e a média da idade motora geral.

A idade motora geral obteve valor mínimo inferior em 23 meses à sua média e valor máximo excedendo em 29 meses ao da média. Vale ressaltar que nenhum dos valores máximo e mínimo das idades específicas de desenvolvimento alcançou a média de suas idades cronológicas.

A média da idade motora da motricidade fina (IM1) foi inferior em 110 meses à média da IC. O valor mínimo dessa variável correspondeu a uma idade inferior em 27 meses ao da média. $\mathrm{O}$ valor máximo foi superior em 27 meses.

A média da idade motora da motricidade global (IM2) foi inferior em 114 meses à média da IC. O valor mínimo dessa variável correspondeu a uma idade inferior em 23 meses ao da média. $\mathrm{O}$ valor máximo foi superior em 37 meses.

A média da idade motora do equilíbrio
(IM3) foi inferior em 117 meses à média da IC. $\mathrm{O}$ valor mínimo dessa variável correspondeu a uma idade inferior em 20 meses ao da média. $\mathrm{O}$ valor máximo foi superior em 40 meses.

A média da idade motora do esquema corporal (IM4) foi inferior em 119 meses à média da IC. O valor mínimo dessa variável correspondeu a uma idade inferior em 18 meses ao da média. $\mathrm{O}$ valor máximo foi superior em 30 meses.

A média da idade motora da organização espacial (IM5) foi inferior em 114 meses à média da $\mathrm{IC}$. $\mathrm{O}$ valor mínimo dessa variável correspondeu a uma idade inferior em 11 meses ao da média. O valor máximo foi superior em 49 meses.

A média da idade motora da organização temporal (IM6) foi inferior em 120 meses à média da IC. O valor mínimo dessa variável correspondeu a uma idade inferior em 17 meses ao da média. $\mathrm{O}$ valor máximo foi superior em 31 meses.

Observando-se a Tabela 1, a média dos escores do quociente motor geral e a média dos quocientes motores específicos da motricidade fina, motricidade global, equilíbrio, esquema corporal, organização espacial e organização tem- 
poral foram representativos do nível "muito inferior", segundo a "EDM". O quociente motor referente à motricidade fina foi o que obteve $o$ menor déficit, sendo que à organização temporal atribuiu-se o maior déficit.

\section{Lateralidade}

Quanto à lateralidade, $40 \%$ da amostra apresentaram lateralidade destra completa, 35\% indefinida, $10 \%$ sinistra e $15 \%$ cruzada.

\section{DISCUSSÃO}

\section{Perfil motor}

Nos estudos de Rosa Neto ${ }^{14}$ com 141 crianças de 3 a 10 anos, do ensino pré-escolar e de $1^{\mathrm{a}}$ a $4^{\mathrm{a}}$ série do ensino fundamental e de Rodrigues ${ }^{15}$ com 106 pré-escolares de 5 e 6 anos, ambos em amostra sem alterações mentais e usando a "EDM", o quociente motor geral foi correspondente a 91 e 102, respectivamente, caracterizando um padrão motor "normal médio".

O quociente motor geral deste estudo, em nível "muito inferior" e de indivíduos sem desordens mentais, mostrados nos estudos supracitados, em padrão "normal médio", indicam a estreita relação entre as dificuldades motoras e as alterações do aspecto mental. Piaget explica a gênese da inteligência mediante a coordenação progressiva dos esquemas sensório-motores ${ }^{16}$, fato reiterado pela insuficiência motora da amostra.

O mesmo instrumento de avaliação deste estudo foi utilizado nas pesquisas de Rosa Neto ${ }^{17}$ com escolares com transtorno de aprendizagem e com alto risco neurológico e de Rosa Neto, Costa e Poeta ${ }^{18}$ também com escolares com problemas de aprendizagem, os quais serão relacionados no decorrer da discussão dos dados.

Neste estudo, a média da idade motora da motricidade fina (IM1) foi de 51 meses, variável que apresentou o maior valor. Em pesquisa de escolares $(n=71)$ entre 3 a 10 anos com transtornos de aprendizagem foi obtida idade média de 72,9 meses para o mesmo teste de motricidade fina ${ }^{17}$. Em uma população $(n=95)$ de 3 a 10 anos com alto risco neurológico, este autor encontrou média da IM1 de 68,7 meses. Entre sujeitos com problemas de aprendizagem entre $5 \mathrm{e}$ 14 anos $(\mathrm{n}=105)$ a média foi de 81 meses $^{18}$.

A partir de movimentos cinemáticos, estudou-se a motricidade fina de 32 crianças de 6 a 10 anos com inabilidade de aprendizagem, confirmando-se a presença de problemas motores nessa população ${ }^{10}$. Bonifacci ${ }^{12}$ encontrou entre 144 crianças da mesma faixa etária diferenças significativas na integração viso-motora entre aquelas com alto e baixo nível de habilidades motoras. Salienta-se que existe relação entre a coordenação óculo-motora e a formação da vida mental da criança ${ }^{16}$.

Foi obtido para a idade da motricidade global (IM2) o valor médio de 47 meses. Em população com transtorno de aprendizagem essa variável teve média de 75,2 meses e 74,3 meses em escolares com alto risco neurológico ${ }^{17}$. Entre escolares com problemas de aprendizagem a média foi de 89 meses $^{18}$.

A relação entre dificuldade de coordenação motora e inabilidade de aprendizagem pode indicar uma vulnerabilidade aumentada dos sistemas neurais responsáveis pela integração da informação sensório-motora ${ }^{19}$. A motricidade global exige a interação entre a tonicidade e a equilibração, além da coordenação da lateralidade, da noção do corpo e da estruturação espaçotemporal, harmonizando assim o espaço intracorporal com o extracorporal ${ }^{16}$.

$\mathrm{O}$ valor médio encontrado para a idade motora do equilíbrio (IM3) foi de 44 meses. Em população com transtornos de aprendizagem, a média da IM3 foi de 54,3 meses e 57,3 meses entre escolares com alto risco neurológico ${ }^{17}$. A média de 73,1 meses foi achada entre crianças e adolescentes com problemas de aprendizagem ${ }^{18}$.

Em estudo sobre o equilíbrio de crianças entre 6 e 12 anos, foi achado que aquelas com dificuldades de coordenação não mantêm o equilíbrio na postura bípede com eliminação momentânea da visão, evidenciando o desequilíbrio motor $^{20}$. Testes motores geralmente demonstram que crianças com atraso no desenvolvimento ou deficiências no domínio perceptual-motor têm equilíbrio insuficiente ${ }^{20}$. 
A insuficiência do equilíbrio motor pode indicar disfunção cerebelar ${ }^{20}$. O cerebelo é essencial para o controle motor e sua disfunção intervém no equilíbrio, fala, coordenação dos membros e olhos, organização espacial, resultando em atraso no desenvolvimento motor ${ }^{6,7,20}$.

A idade motora do esquema corporal (IM4) obteve neste estudo média de 42 meses. Em estudo de escolares com transtorno de aprendizagem, a média da IM4 foi de 71,7 meses e 66,3 meses entre população de alto risco neurológico ${ }^{17}$. A média de 81,1 meses foi achada entre sujeitos com problemas de aprendizagem ${ }^{18}$.

Esta organização é o ponto de partida das possibilidades de ação tendo papel fundamental no desenvolvimento da criança. Por conseguinte, a aquisição dos marcos motores como o controle cervical e do tronco, mobilidade de membros superiores que, a exemplo, permitem que a criança se mantenha na posição sentada e se relacione com o meio, são fundamentais à esquematização do corpo. Em deficientes mentais esta autonomia, por exigir grau elevado de maturação, muitas vezes está ausente ou se apresenta de forma insuficiente.

$\mathrm{O}$ achado referente à média da idade motora da organização espacial (IM5) foi de 47 meses. Em estudo com escolares com problemas de aprendizagem, 76,2 meses foi a média encontrada $^{18}$

Em estudos referentes à inteligência e atividades espaço-temporais, indivíduos com índices elevados de inteligência (QI = 124) apresentaram atividade elétrica cortical elevada referente a esta habilidade, quando submetidos à resolução de problemas ${ }^{21}$. Este fato confirma a associação de inteligência e organização espaço-temporal. Entre os déficits no processamento de informações, os relacionados a modalidades visoespaciais são os mais pronunciados entre indivíduos com inabilidade de aprendizagem ${ }^{22}$.

A percepção da direção, do espaço e da forma pode ser reforçada pelo sentido cinestési$\mathrm{co}^{23}$. A escassez de capacitação motora que acompanha o desenvolvimento da criança deficiente mental não lhe permite integrar dados sensitivos e perceptivos do ambiente e estabelecer relações físicas entre os objetos no espaço e o próprio corpo. Ajustes posturais são basicamente controlados por parâmetros espaciais, referentes à especificidade de direção e temporais, no que tange ao envolvimento oportuno de atividade muscular ${ }^{24}$.

A idade motora da organização temporal (IM6) desta pesquisa obteve média de 41 meses. Os valores de 68,1 e 67,6 meses foram encontrados para as médias da IM6 em, respectivamente, escolares com transtornos de aprendizagem e alto risco neurológico ${ }^{17}$. Entre crianças e adolescentes com problemas de aprendizagem a média foi 66 meses $^{18}$.

Tem sido reconhecido que alterações no processamento temporal podem ser atribuídas a sinais cognitivos e motor-perceptuais, comumente associados a desordens no aprendizado ${ }^{25}$. Além disso, crianças com essas desordens têm capacidade limitada de assimilar informações e precisam de mais tempo para realizá-las em um contexto de atividades motoras ${ }^{10}$.

Elucida-se que a noção do tempo, por requerer a percepção e o entendimento de mudanças de velocidade, espaço recorrido, movimento, crescimento de medida, entre outros atributos físicos, exige uma elaboração eficiente de nossas informações sensoriais, interpretadas e armazenadas no córtex cerebral, onde são processados todos e mais complexos tipos de informação sensorial ${ }^{26}$. Em especial, os sinais do sistema sensorial somático estão localizados no lobo parietal, região que deve manter-se intacta para a adequada elaboração da organização temporal e também espacial ${ }^{27}$, processadas principalmente no hemisfério cerebral direito ${ }^{9,23}$.

\section{Lateralidade}

A maior freqüência de lateralidade destra completa encontrada neste estudo corrobora com pesquisa entre escolares com transtornos de aprendizagem $(\mathrm{n}=71)$, com alto risco neurológico $(n=95)$, com transtorno fonológico $(n=24)$, em que se obteve, respectivamente, $42,3 \%, 50,5 \%$ e $54,2 \%$ de crianças com esta definição de lateralidade ${ }^{14}$ e também com o estudo de escolares com problemas de aprendizagem $(n=105)$ em que $41,9 \%$ da amostra foi destra 
completa $^{18}$. Autores referem o predomínio da lateralidade destra ao citar a existência de $90 \%$ das pessoas com esta preferência ${ }^{23}$. A lateralidade foi estudada entre 17 fetos humanos durante o $1^{\circ}$ e $2^{\circ}$ trimestre gestacional e $83,3 \%$ deles demonstraram mais movimentos à direita ${ }^{28}$.

Certos padrões de comportamento prénatal são atribuídos ao estabelecimento e formação de conexões neurais no sistema nervoso central ${ }^{29}$, relacionando a ontogênese do comportamento da lateralidade à maturação de estruturas cerebrais ${ }^{28}$. Todavia, embora o comportamento motor assimétrico no período embrionário seja mediado pela genética, outros fatores também podem ter importante função na expressão da lateralidade, como condições patológicas ${ }^{28}$.

A lateralidade não definida pode resultar em problemas de aprendizagem ${ }^{16}$. Ocorre que, no caso de deficientes mentais, os problemas de aprendizagem que acompanham essa população podem ser os agentes da lateralidade indefinida, encontrada em $15 \%$ de casos neste estudo. Por outro lado, essa definição pode ter sido atribuída ao inadequado desenvolvimento de assimetrias cerebrais, estruturais e funcionais, de crianças com DM.

\section{CONCLUSÕES}

Os dados da avaliação motora demonstraram, de maneira quantitativa, que deficientes mentais de grau moderado possuem alterações da motricidade. As habilidades avaliadas, motricidade fina e global, equilíbrio, esquema corporal, organização espacial e temporal foram classificadas em padrão motor "muito inferior", sendo a motricidade fina a variável de maior quociente de desenvolvimento entre as habilidades estudadas e a organização temporal a de menor quociente. Verificou-se na literatura a escassez de estudos que relacionam deficiência mental e habilidades motoras específicas.

A educação psicomotora, quando estimulada desde a tenra idade, pode amenizar os déficits mentais das crianças com este transtorno. As instituições que assistem a crianças portadoras de deficiência mental, por meio da observação das condutas motoras durante o desenvolvimento infantil, possibilitam a reabilitação precoce na presença de desvios evolutivos que, se forem desconsiderados, podem se tornar incapacitantes ao indivíduo.

\begin{abstract}
The motor control of mentally retarded persons is altered by the insufficient information between afferent and efferent commands. The purpose of this study was to evaluate the motor profile of children and adolescents with moderate mental retardation. The study group was composed of 20 male and female children and adolescents between 7 and 15 years old, students attending the APAEs (Association of Parents and Friends of the Handicapped) at the cities of Biguaçu and Palhoça, state of Santa Catarina. Data were collected by means of the Motor Development Scale "MDS"14. Statistical treatment was made by Epi. Info 6.0. The level of all the motor abilities was evaluated as "very inferior". The fine motricity quotient got the lowest deficit while the quotient of temporal organization obtained the highest deficit. These results showed the close relation between the cognitive potential and the motor conduct of persons with moderate mental retardation. Their functional deficits can be a consequence of their inadequate psychomotor abilities, interfering in cognitive, affective and social development.
\end{abstract}

Key words: Motor profile. Children. Adolescents. Moderate mental retardation.

\section{REFERÊNCIAS}

1. Croen LA, Grether JK, Selvin S. The epidemiology of mental retardation of unknown cause. Pediatrics. 2001;107(6):86-90.

2. Chechlacz M, Gleeson JG. Is mental retardation a defect of synapse structure and function? Pediatr Neurol. 2003;29(1):11-7.

3. Battaglia A, Carey JC. Diagnostic evaluation of developmental delay/mental retardation: an overview. Am J Med Genet. 2003;117(1):3-14.

4. Panek PE, Smith J. Assessment of terms to 
describe mental retardation. Res Dev Disabil. 2005;26(6):565-76.

5. Kaufmann W, Moser H. Dendritic abnomalies in disorders associated with mental retardation cerebral cortex. Cereb Cortex. 2000;10(10):981-91.

6. Rosenbaum DA, Carlson RA, Gilmore RO. Acquisition of intellectual and perceptualmotor skills. Annu Rev Psychol. 2001;52:453-70.

7. Schweizer K, Koch W. Perceptual processes and cognitive ability. Intelligence. 2000;31(3):211-35.

8. Casey BJ, Giedd JN, Thomas KM. Structural and functional brain development and its relation to cognitive development. Biol Psychol. 2000;54(1-3):241-57.

9. Hernández-Muela S, Mulas F, Mattos L. Plasticidad neuronal funcional. Rev Neurol. 2004;38:58-68.

10. Smits-Engelsman BCM, Wilson PH, Westenberg Y, Duysens J. Fine motor deficiencies in children with developmental coordination disorder and learning disabilities: an underlying open-loop control deficit. Hum Mov Sci. 2003;22(4-5):495-513.

11. Schmitz C, Assaiante C. Development sequence in the acquisition of anticipation during a new co-ordination in a bimanual load-lifting task in children. Neurosci Lett. 2002;330(3):215-8.

12. Bonifacci P. Children with low motor ability have lower visual-motor integration ability but unaffected perceptual skills. Hum Mov Sci. 2004;23(2):157-68.

13. American Psychiatric Association. Diagnostic and statistical manual of mental disorders. 4th ed. Washington (DC); 1994.

14. Rosa Neto F. Valoracion del desarrollo motor y su correlacion con los transtornos del aprendizage [tesis]. Zaragoza: Ciencias de la Actividad Fisica y el Deporte de la Facultad de Medicina de la Universidad de Zaragoza; 1996.

15. Rodrigues LR. Caracterização do desenvolvimento físico, motor e psicossocial de pré-escolares de Florianópolis - SC [dissertação]. Florianópolis: Centro de Educação Física, Fisioterapia e Desportos da Universidade do Estado de Santa Catarina;2000.

16. Fonseca V. Manual de observação psicomotora: significação psiconeurológica dos fatores psicomotores. Porto Alegre: Artes Médicas; 1995.

17. Rosa Neto F. Manual de avaliação motora. Porto Alegre: Artmed; 2001.

18. Rosa Neto F, Costa SH, Poeta LS. Perfil motor em escolares com problemas de aprendizagem. Pediatr Mod. 2005;41(3):109-17.

19. Waber DP, Weiler MD, Bellinger DC, Marcus DJ, Forbes PW, Wypij D, et al. Diminished motor timing control in children referred for diagnosis of learning problems. Dev Neuropsychol. 2000;17(2):181-97.

20. Geuze RH. Static balance and developmental coordination disorder. Hum Mov Sci. 2003;22(45):527-48.

21. Jausovec N, Jausovec K. Spatiotemporal brain activity related to intelligence: a low resolution brain electromagnetic tomography study. Cogn Brain Res. 2003;16(2):267-72.

22. Wilson PH, Maruff P, Ives S, Currie J. Abnormalities of motor and praxis imagery in children with developmental coordination disorder. Hum Mov Sci. 2001;20(1-2):135-59.

23. Vogel J, Bowers CA, Vogel D. Cerebral lateralization of spatial abilities: a meta-analysis. Brain Cogn. 2003;52(2):197-204.

24. Fits IBM, Hadders-Algra M. The development of postural response patterns during reaching in healthy infants. Neurosci Biobehav Rev. 1998;22(4):521-6.

25. Habib M. The neurological basis of developmental dyslexia: an overview and working hypothesis. Brain. 2000;123 Pt 12:237399.

26. Bear M, Connors B, Paradiso M. Neuroscience: exploring the brain. Maryland: Williams \& Wilkins; 1996.

27. Becchio C, Bertone C. Object temporal connotation. Brain Cogn. 2003;52(2):192-6.

28. McCartney G, Hepper P. Development of lateralized behavior in the human fetus from 12 to 27 weeks'gestation. Dev Med Child Neurol. 1999;41(2):83-6.

29. Reigger-Krugh C. Relationship of mechanical and movement factors to prenatal musculoesqueletal development. Phys Occup Ther Pediatr. 1993;12:19-36.

Recebido em: 05/03/2006

Modificado em: 19/09/2006

Aprovado em: 25/09/2006 